\title{
Magnetic softening of Young's modulus of amorphous $\mathrm{Fe}_{90} \mathrm{Zr}_{10}$.
}

\author{
S. H. Park, Y. H. Jeong甘 \\ Department of Physics, Pohang University of Science and Technology \\ Pohang, 790-784, S. Korea \\ K. Nahm, C. K. Kim \\ Department of Physics, Yonsei University, Seoul, 120-749, S. Korea
}

(October 7, 2018)

\begin{abstract}
The Young's modulus and the internal friction of amorphous $\mathrm{Fe}_{90} \mathrm{Zr}_{10}$ alloy were measured near the Curie temperature using the vibrating reed technique. The modulus shows softening around $T_{c} \approx 227 K$ and the internal friction undergoes drastic increase at $T_{c}$. It is found that both the Young's modulus and the reciprocal of internal friction are inversely proportional to the magnetic susceptibility in the paramagnetic phase.
\end{abstract}

PACS numbers: 75.50.K, 62.20.D, 75.80

Typeset using REVTEX 
Amorphous iron-rich Fe-Zr alloys near 90 at. \% Fe have received considerable attention recently because of their unusual magnetic properties. These alloys have been extensively studied by various methods such as magnetic, [1 3] thermal, [2, [1] transport, [4 6 Mössbauer, [3]:7] and neutron scattering [8] measurements. From these measurements, [2] it is known that the system undergoes two successive transitions from a paramagnetic to ferromagnetic at the Curie temperature $\mathrm{T}_{c}$ (with $\mathrm{T}_{c}$ decreasing with increasing iron concentration above 85 at. $\% \mathrm{Fe})$ and to a spin glass phase at $\mathrm{T}_{f}\left(\right.$ less than $\left.\mathrm{T}_{c}\right)$. However, the exact nature of these transitions and magnetic states is still in controversy. [1,2]

Of particular interest about these alloys is its temperature dependence of magnetoelastic properties. It was reported that the a-Fe${ }_{90} \mathrm{Zr}_{10}$ shows the Invar anomaly in the thermal expansion [9]. Despite the fact that many magnetic alloys have been shown to be of Invar type since the first discovery by Guillaume, 10 the physical mechanism of the effect has never been fully understood and still is the subject of active research. Although the name 'Invar' originated from an anomaly in the thermal expansion, Invars are usually found to have a broad variety of associated anomalies. [11] A large magnetic softening in the elastic modulus, for example, frequently accompanies the thermal expansion anomaly near the Curie temperature. [12] Since most of the works on the magnetoelastic properties of Invar alloys have been done on crystalline alloys, 11, 13 it is of value to study the elastic properties of $\mathrm{a}^{-\mathrm{Fe}_{90}} \mathrm{Zr}_{10}$ which is an amorphous invar. This in turn would shed light on the magnetic nature of the system. In this paper, we report the results of our investigation on the variation of the Young's modulus of a- $\mathrm{Fe}_{90} \mathrm{Zr}_{10}$ as a function of temperature.

A frequently used method to measure the elastic modulus of solids is ultrasonic measurements. However, this method is not suitable for a-Fego $\mathrm{Zr}_{10}$ due to the fact that samples are of ribbon type as is usual with metallic glasses. Instead the vibrating reed technique, 14.15 which is an excellent method for the measurements of the elastic modulus of metallic films, was used to investigate the temperature dependence of the Young's modulus and the internal friction of amorphous $\mathrm{Fe}_{90} \mathrm{Zr}_{10}$ ribbons from $100 \mathrm{~K}$ up to $300 \mathrm{~K}$. Since the details of the experiment will be reported elsewhere, [16] we only briefly describe the sample preparation 
method and the vibrating technique here.

The Fe-Zr alloy ingot 10 at \% Zr was prepared by the arc melting in a purified argon atmosphere. The ribbon, about $2 \mathrm{~mm}$ wide and $20 \mu \mathrm{m}$ thick, was prepared by the rotating drum technique under an argon atmosphere. Small parts of the amorphous ribbon were investigated by x-ray diffraction and found to have x-ray diagrams characteristic of the amorphous state, where sharp diffraction lines indicating the presence of the crystalline phases were absent. No deviation from the nominal concentration within the uncertainty of the measurement of 1 at \% could be detected with microprobe analysis.

In measuring the Young modulus, one end of the sample was clamped between two copper flats and the free end was driven electrostatically by a sinusoidal voltage of frequency $f / 2$. Since in an electrostatic drive without bias voltage the resulting force is proportional to the square of the voltage, oscillation occurs at frequency $f$. When $f$ matches with one of the resonant frequencies $f_{n}$ of the ribbon, then resonance occurs. For flexural modes the resonant frequencies are given by [17]

$$
f_{n}=\frac{d}{4 \pi \sqrt{3}}\left(\frac{\beta_{n}}{L}\right)^{2} \sqrt{\frac{E}{\rho}}
$$

where $d$ is the thickness of the ribbon, $L$ its length, $E$ the Young's modulus, and $\rho$ the mass density. $\beta_{n}$ 's are constants which satisfy the equation $\cos \beta_{n} \cosh \beta_{n}+1=0$ and the values of the first few ones are $1.875,4.694,7.855$. The amplitude of the vibration was detected capacitively. The setup of the sample cell is shown in the inset of Fig. 2. As the reed vibrates, the reed and the detecting electrode forms a time varying capacitance whose ac amplitude is proportional to the displacement of the reed. This variation of the capacitance was picked up with a lock-in amplifier. The resonant frequency and the internal friction $Q^{-1}$ were obtained from the shape of the resonance curve by sweeping the driving frequency through the resonance and fitting the data with the damped harmonic oscillator form. During each frequency sweep which took about 5 minutes temperature was controlled with stability of $5 \mathrm{mK}$.

In order to locate the transition point we first carried out the measurements of the 
magnetic susceptibility using the mutual inductance ac-method. [18] An oscillating magnetic field of less than $5 \mathrm{mOe}$ at $48 \mathrm{~Hz}$ was applied along the length of a $1 \mathrm{~cm}$ long sample to minimize demagnetizing effects. Temperature was stable at a given set point within $5 \mathrm{mK}$. Fig. 1 is the plot of the susceptibility against temperature. As temperature falls toward $T_{c}, \chi$ rises sharply and goes through a maximum called a Hopkinson peak. Fitting the data of the paramagnetic phase with the usual power law, $\chi \sim\left|T-T_{c}\right|^{-\gamma}$, yields $\mathrm{T}_{c}=227 \mathrm{~K}$ and $\gamma=$ 1.39. These values are in good accordance with previous measurements. [19] Notice that the data deviate from the fit in the vicinity of $T_{c}$. This is due to the unavoidable demagnetizing effect and one should regard the solid line as representing the intrinsic values.

In Fig. 2 we plot the variation of the Young's modulus of a-Feg0 $\mathrm{Zr}_{10}$, normalized to that at $300 \mathrm{~K}$, as a function of temperature. For this particular sample, the length of the reed was about $1 \mathrm{~cm}$ which resulted in resonance frequencies $f_{1} \simeq 106 \mathrm{~Hz}$ and $f_{2} \simeq 660 \mathrm{~Hz}$ at $300 \mathrm{~K}$. The measurements were done by detecting the fundamental frequency as the temperature was varied. The absolute value of the modulus at $300 \mathrm{~K}$ was calculated, using Eq. (1) to yield $E \simeq 3.5 \times 10^{11}$ dyne $/ \mathrm{cm}^{2}$ which gives the sound velocity $s \simeq 3.3 \mathrm{~km} / \mathrm{s}$. The modulus does not vary appreciably as the temperature is lowered from $300 \mathrm{~K}$ to $240 \mathrm{~K}$ where the sudden softening starts to set in. This abrupt softening continues until the temperature reaches $\mathrm{T}_{c}$. In the ferromagnetic phase the modulus still decreases, with the smaller slope, as the temperature is reduced further. The behavior of the Young's modulus in the ferromagnetic phase represents the interesting physics; however, we defer the discussion of this point to the full paper [16] and restrict our discussion on the paramagnetic phase in this letter.

It is noted that the modulus softening occurs mostly above $\mathrm{T}_{c}$, i.e., in the paramagnetic phase. This behavior cannot be explained in terms of the Landau theory which is frequently invoked to account for magnetic softening of elastic constants. [20] Rather, this result strongly indicates the importance of coupling between the spin fluctuations and phonons. Thus, it would be appropreate to investigate the relationship between the elastic modulus and the magnetic susceptibility which is a measure of the spin fluctuations. Fig. 3 is the plot of the Young's modulus against the inverse of susceptibility. Self-evident is the 
linear relationship between $\mathrm{E}$ and $1 / \chi$ in the temperature range near $\mathrm{T}_{c}$ where most of the softening of $\mathrm{E}$ and the increase in $\chi$ occur. This is, to our knowledge, the first time this feature is displayed in metallic magnetic systems.

Since the modulus is strongly affected by the magnetic phenomenon occuring in the system, it is reasonable to expect that the internal friction would also reflect the magnetic ordering. To see this effect, however, one must take into account the thermoelastic relaxation [21], since it usually is an important loss mechanism in the vibrating reed. According to Golding [14], damping caused by the thermoelastic relaxation is given by

$$
Q_{t h}^{-1}=T E_{T} \alpha_{p}^{2} / C_{p} \frac{\omega \tau}{1+\omega^{2} \tau^{2}}
$$

with $\tau=(d / \pi)^{2} C_{p} / \kappa$ where $d$ is the thickness of the sample. Here $E_{T}, \alpha_{p}, \kappa$ and $C_{p}$ are the isothermal Young's modulus, linear thermal expansivity, thermal conductivity, and isobaric specific heat respectively. Evaluating Eq. (2) by putting the values of relevant parameters, we obtain $Q_{t h}^{-1} \sim 6.5 \times 10^{-8}$. Since this value is very much smaller than those measured experimentally, we can safely disregard thermoelastic relaxation effects. Note that the facts that a-FeZr is an Invar, i.e., $\alpha$ is small, and has metallic thermal conductivity allow the vibrating reed technique to be an ideal method to probe any change in the internal friction due to the intrinsic effect associated with the magnetic ordering. This is to be contrasted to the situation with $\mathrm{EuO}$ where the thermoelastic relaxation is dominant. [14]

The inset of Fig. 4 displays the internal friction as a function of temprature. Indeed there is a steep increase in the internal friction $Q^{-1}$ in conjunction with the softening of the modulus near $\mathrm{T}_{c}$. Remembering that the steep increases are present both in the susceptibility and in the internal friction near $\mathrm{T}_{c}$, we have plotted in Fig. 田 the inverse of the internal friction, $1 / Q^{-1}$, versus the reciprocal of the susceptibility. Here again the linear relationship is obeyed in the same temperature range as was with the Young's modulus and the susceptibility.

At present we are not aware of any theory which is fully capable of explaining our experimental results. However, the theory due to Kim 23 which stresses the role of the 
electron-phonon interaction in metallic magnetism seems to be relevant. According to this theory, the dynamic screening of phonons by the mutually interacting electrons is the main cause of all the anomalies of metallic magnetism; assuming the jellium model for a metal 24] and using the mean field approximation, he showed how the phonon frequency is influenced by the electronic behaviors. The relationship between the velocity and the attenuation of sound and the magnetic susceptibility is especially simple in the paramagnetic phase. That is, the sound velocity s and the sound attenuation $\Gamma$ are given by

$$
\begin{aligned}
s / s_{0} & =\left[\xi+\chi_{0} / \chi\right]^{1 / 2} \\
\Gamma / \Gamma_{0} & =\left(s_{0} / s\right)^{2}
\end{aligned}
$$

where $\chi$ is the Stoner susceptibility and $s_{0}$ is the Bohm-Staver sound velocity of the system. $\chi_{0}, \Gamma_{0}$ and $\xi$ are constants characterizing the system and of these constants $\xi$ is the only parameter which can be temperature dependent. $\xi$ quantifies how much the system deviates from the jellium model which assumes point-like ionic interactions; in other words, it quantifies short-range steric interactions between ions. The wavenumber dependent frequency, $\Omega(q)$, for longitudinal oscillations of ions is given by

$$
\Omega(q)^{2}=\Omega_{p}^{2}+\xi s_{0}^{2} q^{2}
$$

where $\Omega_{p}$ is the ionic plasma frequency. Thus, one expects that it would be a very sensitive function of distance between ions and consequently dominates the temperature dependence of Eq. (3) in ordinary situations. However, for Invars $\xi$ may be regarded as a constant, since $\alpha$ is extremely small.

Thus, assuming that $a-\mathrm{Fe}_{90} \mathrm{Zr}_{10}$ is an itinerant ferromagnetic system, 25] Kim's theory may appear to correctly account for the experimental results since the Young's modulus is proportional to square of the sound velocity and $\xi$ does not vary much within the narrow temperature range near $\mathrm{T}_{c}$ where most of changes in the susceptibility, the sound velocity, and the internal friction occur. However, it should be kept in mind that the susceptibility appearing in the theory is the Stoner one, which does not have the same temperature 
dependence as the measured one. Therefore, it is premature to conclude that the theory is supported by the experimental results. We merely point out that Kim's theory would be more suitable for amorphous metals in view of the fact that the theory is that of free electrons based on the Jellium model, which was pointed out as a weak point of the theory. 26

In conclusion, we have discovered that the Young's modulus and the inverse of the internal friction of a- $\mathrm{Fe}_{90} \mathrm{Zr}_{10}$ alloy are linearly proportional to the reciprocal of the magnetic susceptibility in the paramagnetic phase. This behavior seems to suggest the importance of the electron-phonon interaction in this system. Sytematic study is under way to see whether this effect is universal in amorphous Invars.

We gratefully acknowledge invaluable discussions with Prof. B. I. Min. YHJ wish to thank financial supports from Korea Research Foundation and Dong-il Foundation. This work was partly supported by the BSRI program of POSTECH and Korea-Russia Collaboration program of KOSEF. 


\section{REFERENCES}

* To whom all the correspondence should be addressed.

[1] N. Saita, H. Hiroyoshi, K. Fukamichi and Y. Nakagawa, J. Phys. F: Met. Phys. 16, 911 (1986).

[2] J.M.D. Coey, D.H. Ryan, and R. Buder, Phys. Rev. Lett. 포, 385 (1987).

[3] J.A. Heller, E.F. Wassermann, M.F. Braun and R.A. Brand, J. Mag. Mag. Mater. 54, 307 (1986).

[4] Y. Obi, L.C. Wang, R. Motsay, D.G. Onn and M. Nose, J. Appl. Phys. 므, 2304 (1982).

[5] K. Fukamichi, R.J. Gambino, and T.R. McGuire, J. Appl. Phys. 533, 2310 (1982).

[6] D. Dahlberg, K.V. Raoi and K. Fukamichi, J. Appl. Phys. 55, 1942 (1984).

[7] D.H. Ryan, J.M.D. Coey, E.Batalla, Z. Altounian and J.O. Ström-Olsen, Phys. Rev. B $\underline{35}, 8630(1987)$.

[8] J.J. Rhyne and G.E. Fish, J. Appl. Phys. 57, 3407 (1985).

[9] K. Shirakawa, S. Ohnuma, M. Nose and T. Masumoto, IEEE Trans. Mag. 16, 910 (1980).

[10] Ch. E. Guillaume C. R. Acad. Sci. 125, 235 (1897).

[11] E.F. Wasserman , Ferromagnetic materials Vol. 5, edited by K.H.J. Buschow and E.P. Wohlfarth (Elsevier, Amsterdam, 1990).

[12] E.P. Wohlfarth, J. Phys. F: Met. Phys. ㅁ, L59 (1976).

[13] U. Kawald, P. Schulenberg, H. Bach, J. Pelzl, G. Eckod and G.A. Saunders, J. Appl. Phys. $\underline{70}, 6537$ (1991).

[14] M. Barmatz and B. Golding, Phys. Rev. B 모, 3064 (1974). 
[15] J. Kober, A. Gupta, P. Esquinazi and H.F. Braun, Phys. Rev. Lett. $\underline{66}, 2507$ (1991).

[16] S. H. Park, D. J. Bae, Y. H. Jeong, to be published.

[17] L. E. Kinsler, A. R. Frey, A. B. Coppens, and J. V. Sanders, Fundamentals of acoustics, 3rd ed. (John Wiley \& sons, New York, 1982).

[18] I. K. Moon, Magnetic phase transition of $a-F e_{90} Z r_{10}$, M. S. Thesis, Pohang University of Science and Technology (1992).

[19] S.N. Kaul, J. Phys. F: Met. Phys. 18, 2089 (1988).

[20] M. Shiga, K. Makita, K. Uematsu, Y. Muraoka, and Y. Nakamura, J. Phys.: Condens. Matter $\underline{2}, 1239$ (1990).

[21] C. Zener, Phys. Rev. 모, 230 (1937); Phys. Rev. 푸, 90 (1938).

[22] Y. H. Jeong, S. H. Park, I. K. Moon, D. J. Bae, and K. Nahm, to be published.

[23] D. J. Kim, Physics Reports 171, 129 (1988).

[24] D. Pines, Elementary Excitations in Solids (Benjamin, New York, 1964).

[25] S. N. Kaul, Phys. Rev. B27, 6923 (1983); J. S. Kang, K. Nahm, and C. K. Kim, preprint.

[26] E. P. Wohlfarth, Physica 119B, 203 (1983). 


\section{FIGURES}

FIG. 1. Plot of the susceptibility of a-Feg0 $\mathrm{Zr}_{10}$ against temperature. The solid line represents the power law fit to $\chi \sim\left|T-T_{c}\right|^{-\gamma}$, with $\mathrm{T}_{c}=227 \mathrm{~K}$ and $\gamma=1.39$.

FIG. 2. Temperature dependence of the Young's modulus. The modulus was normalized to the value $\left(\mathrm{E}_{0}\right)$ at $300 \mathrm{~K}$. Inset shows the capacitive cell used in the present measurements.

FIG. 3. Plot of Young's modulus versus $1 / \chi$. The straight line is the guide to the eye.

FIG. 4. Plot of $1 / Q^{-1}$ versus $1 / \chi$. Inset displays $Q^{-1}$ as a function of temperaature. 